\title{
VARIACIÓN TEMPORAL DE LA ABUNDANCIA Y DIVERSIDAD DE AVES LIMÍCOLAS EN EL REFUGIO DE VIDA SILVESTRE PANTANOS DE VILLA, LIMA - PERÚ
}

\section{TEMPORAL VARIATION IN THE ABUNDANCE AND DIVERSITY OF SHOREBIRDS AT THE PANTANOS DE VILLA WILDLIFE REFUGE, LIMA PERU}

\author{
Miriam Torres $^{1}$, Zulema Quinteros ${ }^{2}$ y Fernando Takano ${ }^{1}$
}

\section{Resumen}

En el Refugio de vida silvestre Pantanos de Villa entre Marzo de 2005 a Febrero de 2006 se registró 18 especies de aves limícolas entre migratorias y residentes. Durante la época no reproductiva se registraron dos picos de abundancia en los meses de Setiembre y Enero, siendo este último el mayor. Calidris pusilla, Tringa flavipes y Charadrius semipalmatus fueron las especies que reportaron el mayor número de individuos para Enero lo que sugiere que poblaciones de estas especies provenientes de otros humedales utilizarían a Pantanos de Villa como punto de parada dentro de sus rutas migratorias hacia sus lugares de reproducción. Así mismo, Enero registró crías de las aves residentes Himantopus mexicanus, Charadrius vociferus y Haematopus palliatus lo que repercutió en la alta abundancia registrada en este mes. La diversidad obtenida presentó sus valores más altos en los meses con mayor abundancia

Palabras clave: aves limícolas, aves migratorias, Pantanos de Villa, abundancia, diversidad

\begin{abstract}
From March 2005 to February 20006, 18 species of shorebirds were recorded at the Pantanos de Villa WildLife Refuge, including both resident and migratory species. During the nonbreeding season there were two abundance peaks in September and January, the latter with highest abundance than the former one. Calidris pusilla, Tringa flavipes and Charadrius semipalmatus were species that recorded the highest numbers in January. This suggests that populations of these birds that come from other wetlands will use Pantanos de Villa as a stopover on their migration routes to their breeding places. January also recorded young Himantopus mexicanus, Charadrius vociferus and Haematopus palliates, which increase bird abundance in this month. Diversity results present the highest values in the months that had the highest abundance
\end{abstract}

Key words: shorebirds, migration birds, Pantanos de Villa, abundance, diversity

\section{Introducción}

Los humedales se encuentran entre los ecosistemas más productivos del mundo (Kusler et al., 1994; Pulido, 1998) y constituyen uno de los refugios más importantes para la vida silvestre. Así mismo, los diversos beneficios que éstos proveen a humanidad tales como la pesca, ganadería, turismo entre otros, derivan en un papel central dentro de las estrategias de desarrollo socioeconómico sostenible (RAMSAR, 2002; Tabilo, 2003).

En la región desértica de la costa del Perú, los humedales adquieren gran importancia al constituirse en verdaderos oasis para el paso de las aves migratorias así como sinónimo de supervivencia de las comunidades de aves acuáticas residentes (Pulido, 2003). En el Perú existen 4 áreas naturales protegidas por el estado que por su cercanía al litoral marino son fuertemente influenciados por él; de éstas el Refugio de Vida Silvestre Pantanos de Villa es la única área que se encuentra en una zona urbana, en Lima (Pulido et al., 1996).
El Refugio de Vida Silvestre Pantanos de Villa se encuentra ubicado en el distrito de Chorrillos, Provincia de Lima (INRENA, 1998). En el año 1943 los pantanos presentaban una extensión de 1530 ha (Pulido, 2003). Como consecuencia del crecimiento urbano de Lima, problemas de contaminación y desecación, éstos se han reducido presentando actualmente un área no mayor a 396 ha (Pulido, 2003).

Las aves han sido identificadas como organismos adecuados para ser utilizados como indicadores biológicos o biomonitores de los cambios ambientales en los diferentes tipos de habitas que existen (Eiseimann, 2006; Hollamby et al., 2006). En el caso de las aves acuáticas, esto se fundamenta en que debido a su posición en la cadena trófica éstas se verán afectadas por una gran variedad de factores, tales como la pérdida de hábitat dada por la desecación y degradación de los humedales, así como las diferentes condiciones físicas de los humedales 
que afectan directamente en su abundancia y diversidad (Green \& Figuerola, s/f).

Dentro del grupo de aves acuáticas se encuentran las aves limícolas también conocidas como chorlos, playeros o aves de orilla. Las aves limícolas son aquellas que se alimentan en zonas de limo y/o orillas de mar. Taxonómicamente, éstas se ubican dentro del orden Charadriiforme siendo las familias Haematopodidae, Charadriidae, Scolopacidae, Recurvirostridae y Phalaropodidae las que presentan especies netamente limícolas (Koepcke, 1964; Canevari et al., 2001).

La dieta de las aves limícolas está constituida principalmente de invertebrados macrobentónicos, como insectos de los órdenes Coleoptera, Homoptera, Diptera y de las familias Corixidae (Hemiptera, Heteroptera), entre otros, sin embargo la mayoría de estudios concuerdan que los Chironomidae (Diptera) son su principal alimento (Rundle, 1982; Baldassarre \& Fischer, 1984; Eldridge, 1992; Lyons \& Haig, 1997). Estas aves, dependen directamente de las orillas de mar y zonas de limo ya que estos lugares les proveen su alimento. Sin embargo, características morfológicas de cada familia determinan su lugar de alimentación y técnicas de captura de las presas, pudiendo ser esta caza táctil o visual (Barbosa \& Moreno, 1999).

Otra característica importante en estas aves, es que muchas de ellas son aves migratorias que siguen rutas de migración fijas y constantes a lo largo del tiempo (Myers et al., 1985; Castro et al., 1988; Berthold, 1993). Así, se ha determinado que en el Hemisferio Occidental éstas se reproducen durante los meses de Junio a Agosto, principalmente en las Islas del Ártico Canadiense y Alaska (Castro \& Myers, 1987). En el periodo no reproductivo, se les encuentra en las playas desde los $50^{\circ} \mathrm{N}$ del Océano Pacifico y $35^{\circ} \mathrm{N}$ de la costa Atlántica hasta los $50^{\circ} \mathrm{S}$ (Myers et al., 1985).

Las aves migratorias necesitan de grandes cantidades de energía para realizar sus vuelos. Si ésta no es obtenida con suficiencia en su lugar de arribo, entonces los sitios de descanso son determinantes para su supervivencia por ser estos los que les proveen el alimento (Lyons \& Haig, 1997; Blanco, 2000). A pesar de que Pantanos de Villa ha sufrido una considerable reducción de su área total, este humedal sigue siendo un lugar importante para las aves que hacen uso de él, tanto residentes como migratorias (Guillen \& Barrio, 1994; Wust et al., 1994; Pautrat \& Riveros, 1998; Pulido, 2003). De las 155 especies de aves reportadas para los Pantanos de Villa, 28 especies son aves limícolas de las cuales 24 son migratorias (Wust et al., 1994).

Este trabajo presenta un análisis de la variación temporal de la abundancia y diversidad de las aves limícolas del Refugio de Vida Silvestre Pantanos de Villa entre Marzo del 2005 y Febrero del 2006, así como la determinación de las áreas de mayor importancia para éstas. El conocimiento de estos aspectos podrá ser usado para la protección y conservación de éstas aves y las zonas que ellas usan.

\section{Materiales y métodos}

\section{Lugar de estudio}

En la parte suroeste del Refugio de Vida Silvestre Pantanos de Villa, se trabajó en las áreas de la zona limosa de la laguna La Herradura ( $12^{\circ} 13^{\prime} 05.0^{\prime}$ ' $-12^{\circ}$ 13' 14.6' S; 76 59' 08.2', - 76 58' 53.5', W), en la orilla de la laguna Marvilla $\left(12^{\circ} 13^{\prime} 20.6\right.$ ', $-12^{\circ} 13^{\prime}$ 32.7' 'S; 7659' 20.2'’ $76^{\circ}$ 59' $05.1 \mathrm{~W}$ ) y en la orilla de playa ubicada al oeste de la laguna Marvilla (Figura1). Los nombres utilizados para ambas zonas de estudio fueron proporcionados por el Centro de Interpretación Pantanos de Villa.

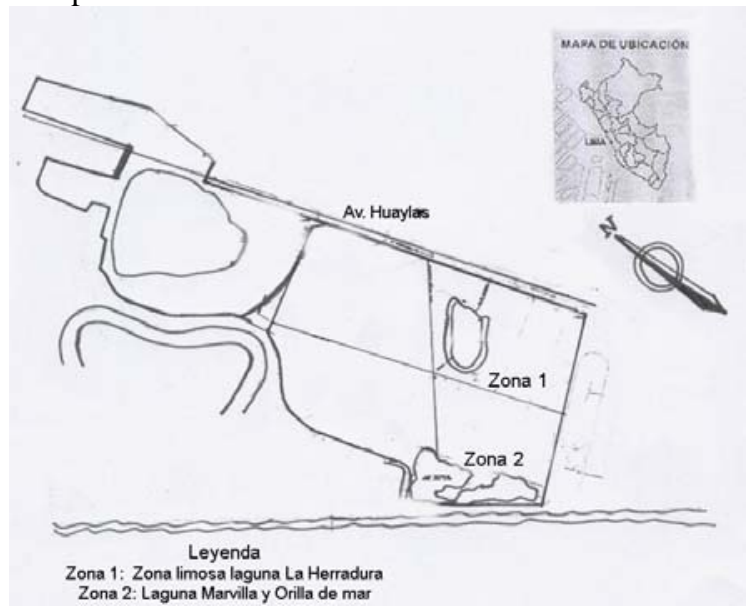

Figura 1. Ubicación de las zonas muestreadas en el Refugio de Vida Silvestre Pantanos de Villa

La zona limosa de la laguna La Herradura se caracteriza por presentar una extensión que varia entre 7 - 12 ha. Esta variación se da como consecuencia de la fluctuación del nivel del agua el cual registra sus mayores niveles (15-40 cm.) en los meses de Junio Septiembre, descendiendo gradualmente entre los meses de Octubre - Marzo, exponiendo así zonas de limo ideales para las aves limícolas. Esta oscilación en los niveles del agua estaría directamente relacionada con los niveles del agua subterránea (Torres, 2006) y no necesariamente con el nivel de agua presente en la laguna La Herradura como señalan Pautrat \& Riveros (1998) y Pulido (2002).

El borde de la laguna Marvilla y la orilla de mar comprendida dentro del Refugio de Vida Silvestre Pantanos de Villa tiene una extensión de 17 ha. El borde de la laguna es arenoso, por lo que la presencia de "totora" (Typha domingensis) es mínima. El nivel del agua presenta una ligera variación de unos pocos centímetros, pero a pesar de ello no afecta a las aves limícolas debido a que al ser el sustrato arena, el borde expuesto no ofrece una nueva área de alimentación (Torres, 2006). Al estar esta laguna a unos pocos 
metros de la orilla de mar y presentar ambas el mismo sustrato, se les considero como una sola zona de muestreo.

Estas áreas se seleccionaron en base a la información disponible de los censos mensuales realizados por SERPAR entre los años 1996 y 2000. En estos censos, estas áreas seleccionadas presentaron las mayores bandadas de aves limícolas (inf. no publicada).

\section{Metodología}

Para el censo de las aves limícolas se utilizó el método del conteo total en ambas zonas evaluadas. Se optó por este método debido a los tamaños de las áreas censadas y por ser estas fácilmente visibles. Para la observación e identificación de las aves se utilizó binoculares (10 x 50) y guías de campo (Fjeldsa \& Krabbe, 1990; Canevari et al., 2001; Clements \& Shany, 2001), teniendo una distancia máxima de observación de $100 \mathrm{~m}$. En cada conteo se registraron: especie, número de individuos y, en algunos casos, evidencias de reproducción en el área. En ambas zonas muestreadas se llevaron a cabo censos mensuales a lo largo de un año con 4 repeticiones por cada lugar, 2 en la mañana (7:45 - 9:00 hr.) y 2 en la tarde (14:0015:00 hr), cada uno con una duración promedio de 40 minutos para determinar la existencia de diferencias de abundancias entre horas muestreadas. El día escogido a censar fue aleatorio entre las dos primeras semanas del mes. Para determinar si el día escogido a censar era representativo del mes, se censó cuatro días seguidos con dos repeticiones, una en la mañana (7:45 h) y una en la tarde (14:00 h), ello con el objetivo de establecer la posible variabilidad de abundancia entre ellos. La comparación de la variación de la abundancia entre los cuatro días muestreados, al igual que la comparación de la abundancia entre las cuatro horas muestreadas a lo largo de un año, se realizó con el análisis ANOVA de Bloques Completamente al Azar (BCA) con un nivel de significación de 0.05. Así mismo, se estimó la diversidad utilizando el índice de Shannon y Wiener (Magurran, 1988).

$$
H=-\sum p_{i} \log _{2} p_{i}
$$

Donde: $\mathrm{pi}=$ proporción de individuos pertenecientes a la $i$ - esima especie.

\section{Resultados y discusión}

Especies de aves registradas

Se registraron 18 especies pertenecientes a 5 familias, del orden Charadriiformes (Tabla 1).

Análisis de Varianza (ANOVA)

Los resultados obtenidos de este análisis demuestran que no existe diferencia significativa entre los días muestreados en ambas zonas evaluadas (laguna La Herradura $\mathrm{F}=12.1 \mathrm{CV}=2.3 \%$; laguna Marvilla y orilla de mar F=7.1 CV 9.8\%), por lo que el día escogido a censar puede ser considerado representativo del mes en que se tomó.

Así mismo, se obtuvo que no existe difererencia significativa en la abundancia de aves entre las cuatros horas muestreadas (laguna La Herradura $\mathrm{F}=1.9$; laguna Marvilla y orilla de mar $F=1.1$ ), por lo que se promedio los cuatro datos de abundancia obteniéndose un solo valor por mes.

Tabla 1. Lista de aves registradas en la Zona limosa de la Laguna la Herradura y Laguna Marvilla

\begin{tabular}{|c|c|c|c|c|}
\hline Familia & Especie & $\begin{array}{l}\text { Nombre } \\
\text { común }\end{array}$ & Categoría & Ubicación \\
\hline Haematopodidae & $\begin{array}{l}\text { Haematopus } \\
\text { palliatus }\end{array}$ & ostero común & $\mathrm{R}$ & 2 \\
\hline Charadriidae & $\begin{array}{l}\text { Pluvialis } \\
\text { squatarola }\end{array}$ & chorlo ártico & Mn & 1.2 \\
\hline Charadriidae & $\begin{array}{l}\text { Charadrius } \\
\text { semipalmatus }\end{array}$ & $\begin{array}{l}\text { chorlo } \\
\text { semipalmado }\end{array}$ & $\mathrm{Mn}$ & 1.2 \\
\hline Charadriidae & $\begin{array}{l}\text { Charadrius } \\
\text { vociferus }\end{array}$ & $\begin{array}{l}\text { chorlo doble } \\
\text { collar }\end{array}$ & $\mathrm{R}$ & 1.2 \\
\hline Scolopacidae & $\begin{array}{l}\text { Arenaria } \\
\text { interpres }\end{array}$ & vuelvepiedras & $\mathrm{Mn}$ & 1.2 \\
\hline Scolopacidae & $\begin{array}{l}\text { Calidris } \\
\text { minutilla }\end{array}$ & $\begin{array}{l}\text { playerito pico } \\
\text { fino }\end{array}$ & $\mathrm{Mn}$ & 1.2 \\
\hline Scolopacidae & $\begin{array}{l}\text { Microplamathus } \\
\text { himantopus }\end{array}$ & $\begin{array}{l}\text { playero pata } \\
\text { larga }\end{array}$ & $\mathrm{Mn}$ & 1 \\
\hline Scolopacidae & Calidris mauri & $\begin{array}{l}\text { playerito } \\
\text { occidental }\end{array}$ & $\mathrm{Mn}$ & 1.2 \\
\hline Scolopacidae & $\begin{array}{l}\text { Calidris } \\
\text { melanotos }\end{array}$ & $\begin{array}{l}\text { playero } \\
\text { pectoral }\end{array}$ & $\mathrm{Mn}$ & 1 \\
\hline Scolopacidae & Calidris pusilla & $\begin{array}{l}\text { playerito } \\
\text { semipalmado }\end{array}$ & Mn & 1.2 \\
\hline Scolopacidae & Calidris alba & $\begin{array}{l}\text { playero } \\
\text { blanco }\end{array}$ & $\mathrm{Mn}$ & 2 \\
\hline Scolopacidae & $\begin{array}{l}\text { Limnodromus } \\
\text { griiseus }\end{array}$ & $\begin{array}{l}\text { becasina } \\
\text { migratoria }\end{array}$ & $\mathrm{Mn}$ & 1 \\
\hline Scolopacidae & Tringa flavipes & $\begin{array}{l}\text { pata amarilla } \\
\text { menor }\end{array}$ & $\mathrm{Mn}$ & 1.2 \\
\hline Scolopacidae & $\begin{array}{l}\text { Tringa } \\
\text { melanoleuca }\end{array}$ & $\begin{array}{l}\text { pata amarilla } \\
\text { mayor }\end{array}$ & $\mathrm{Mn}$ & 1.2 \\
\hline Scolopacidae & $\begin{array}{l}\text { Actitis } \\
\text { macularia }\end{array}$ & $\begin{array}{l}\text { playero } \\
\text { manchado }\end{array}$ & $\mathrm{Mn}$ & 1.2 \\
\hline Scolopacidae & $\begin{array}{l}\text { Numenius } \\
\text { phaeopus }\end{array}$ & $\begin{array}{l}\text { zarapito } \\
\text { trinador }\end{array}$ & $\mathrm{Mn}$ & 1.2 \\
\hline Recurvirostridae & $\begin{array}{l}\text { Himantopus } \\
\text { mexicanus }\end{array}$ & perrito & $\mathrm{R}$ & 1 \\
\hline Phalaropodidae & $\begin{array}{l}\text { Phalaropus } \\
\text { tricolor }\end{array}$ & $\begin{array}{l}\text { falaropo de } \\
\text { Wilson }\end{array}$ & $\mathrm{Mn}$ & 1.2 \\
\hline $\begin{array}{l}\text { Categoría: } \\
\mathrm{R} \text { Residente } \\
\text { Mn Migratoria ne } \\
\text { Ubicación: } \\
1 \text { Zona limosa de } \\
2 \text { Laguna Marvil }\end{array}$ & $\begin{array}{l}\text { ártica } \\
\text { la laguna La Her } \\
\text { la y orilla de mar }\end{array}$ & dura & & \\
\hline
\end{tabular}

Abundancia de las aves limícolas en las zonas evaluadas

En la zona limosa de la laguna La Herradura se registraron 17 especies de las cuales 2 fueron residentes (Tabla 1). En la Figura 2, se observa la variación de las abundancias promedio total de las aves limícolas y migratorias correspondiente a esta zona. A partir de Mayo hasta Agosto el número de 
individuos disminuye presentando sus valores más bajos entre Junio y Julio, 14 y 9 individuos respectivamente. Esto se debe a que en estos meses las aves migratorias se trasladan hacia el Hemisferio norte para reproducirse. Las especies que se registraron en estas fechas fueron Tringa flavipes, Tringa melanoleuca, y Phalaropus tricolor. Castro \& Myers (1987), señalan que en algunas especies de aves limícolas del Hemisferio sur es común el retraso de su partida e incluso que no lleguen a migrar como lo reporta para Calidris alba, en donde los juveniles de esta especie no migran y por lo tanto no se reproducen durante su primer año de vida. Ellos concluyen ésto debido a que la población de juveniles no llega a desaparecer totalmente y no acumulan grasa por lo que no podrían migrar. En nuestro caso ninguno de las especies previamente mencionadas fueron crías, y más bien Phalaropus tricolor presentó algunos individuos con plumaje reproductivo, lo que sugiere que son individuos jóvenes en etapa reproductiva que no llegaron a migrar.

En Septiembre se observa un aumento de la abundancia promedio dado por la llegada de la mayor parte de especies migratorias, que permanecen en esta zona hasta su próxima migración. En Enero se registra un segundo aumento de abundancia promedio mayor que el registrado en Septiembre. Este aumento en la abundancia responde a que en ésta época aparecen las primeras crías de las aves residentes Himantopus mexicanus y Charadrius vociferus, las cuales inician su periodo reproductivo a finales de octubre (obs. personal). Mientras que en el caso de las migratorias Calidris pusilla, Tringa flavipes y Charadrius semipalmatus, son las especies que ostentan un mayor número de individuos en éste mes (Figura 3). Estos resultados sugieren que las poblaciones de estas especies que se encontrarían en otros humedales, al regresar a sus zonas reproductivas en el Hemisferio norte utilizan a Pantanos de Villa como un sitio de parada durante su ruta de migración. Este mismo patrón se repite para algunas especies en Argentina y Colombia, en donde la mayor abundancia de algunas especies migratorias las registran a fines de Marzo hasta mediados de Abril (Blanco, 2000; Guerra, 2004).

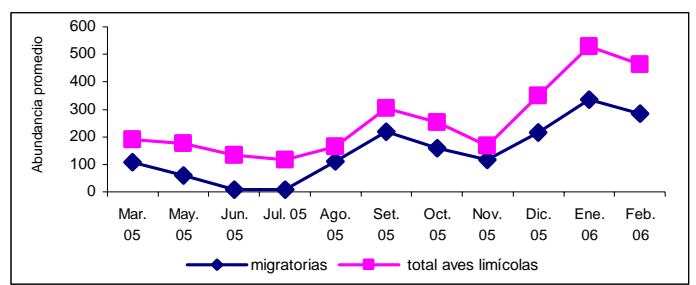

Figura 2. Variación mensual de la abundancia promedio total de aves limícolas y migratorias en la zona limosa de la laguna La Herradura.
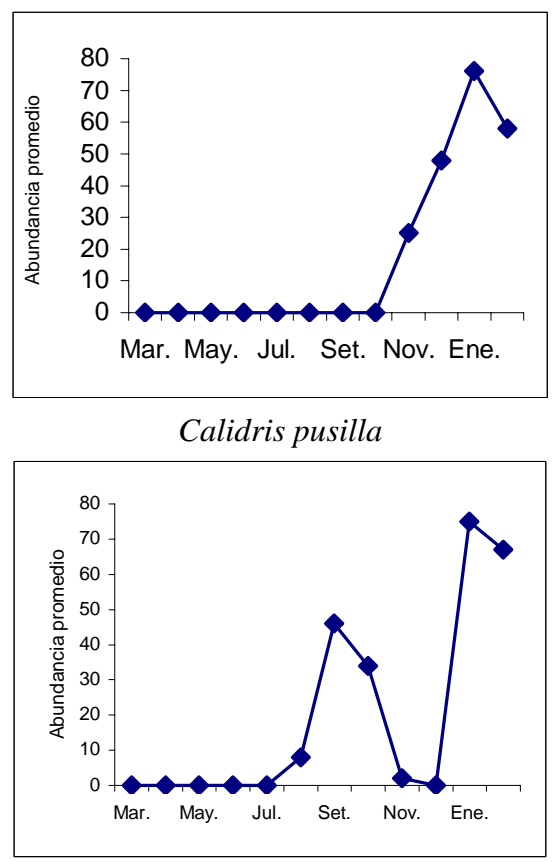

Charadrius semipalmatus

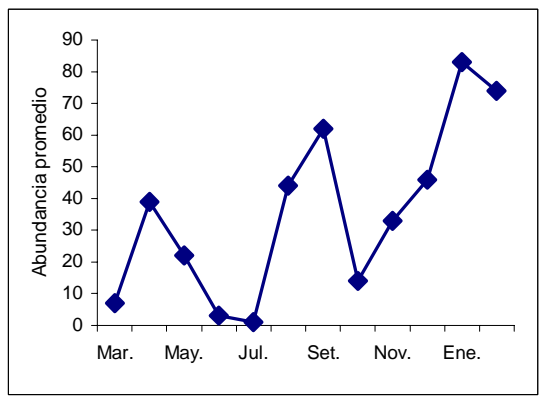

Tringa flavipes

Figura 3. Variación mensual de la abundancia promedio de Calidris pusilla, Charadrius semipalmatus y Tringa flavipes.

Las especies que presentaron las poblaciones más numerosas fueron Himantopus mexicanus, las del género Tringa y algunas del género Calidris, las cuales también presentan la estadía más prolongada en Pantanos de Villa. Especies como Pluvialis squatarola y Calidris melanotos, fueron raramente observadas registrándose 3 y 23 individuos a lo largo del año respectivamente. Una acotación interesante es que Calidris melanotos siempre fue observado en el mismo lugar. Según Reacher \& Reacher (1969) esto se debería a que esta especie es muy territorial y tiende a presentar un comportamiento muy similar en un periodo determinado de tiempo, que puede prolongarse por semanas.

En la laguna Marvilla y la orilla de mar se registraron 14 especies de aves limícolas, siendo 2 de ellas residentes (Tabla 1 ). Si bien es cierto que el número total de especies es muy similar al reportado 
en la zona limosa de la laguna La Herradura, cabe resaltar que la abundancia de éstas es ínfima, salvo algunas excepciones. Esta variación en la abundancia responde al tipo de hábitat que existe en la zona. A pesar de que ambas zonas comparten el mismo tipo de especies, los resultados obtenidos demuestran que la mayor parte de ellas se concentra en la zona limosa debido al tipo de alimentación que presentan. Se llega a esta conclusión debido a los resultados obtenidos del análisis de componentes principales (ACP) realizados entre estas aves y la composición del macrobentos del lugar (Torres, 2006). La mayor parte de las especies de aves limícolas reportadas para esta zona se alimentan principalmente de Coleopteros, Homopteros, Dipteros, Corixidae y en especial de Chironomidae, macrobentos encontrados en zonas limosas (Rundle, 1982; Baldassarre \& Fischer, 1984; Eldridge, 1992). Sin embargo, especies como Calidris alba, Haematopus palliatus, y Pluviales squatarola prefieren orillas de mar donde el "muy muy" (Emerita sp.) es su principal alimento (Castro \& Myers, 1987). Esto demuestra el porque de la preferencia de un hábitat sobre otro por este tipo de aves.

A lo largo del año (Figura 4), la mayor abundancia fue reportada en Setiembre, que corresponde al mes en que llegan las aves migratorias y, además por que fue el mes en el que se registró el mayor número de individuos de Numenius phaeopus y Calidris alba. A pesar que la abundancia promedio de Febrero fue menor que la de Setiembre, cabe resaltar que los valores de abundancia de este mes son mayores a los obtenidos los otros meses evaluados. Este aumento en la abundancia promedio esta dado principalmente por las residentes Charadrius vociferus y Haematopus palliatus, las cuales presentan sus primeras crías en ésta época del año y por Charadrius semipalmatus que al igual que en la zona limosa de la laguna La Herradura presentó el mayor número de individuos. A diferencia de lo reportado para la zona limosa de la laguna La Herradura, la laguna Marvilla sí presento 2 meses, Junio - Julio, en donde hubo ausencia total de aves migratorias.

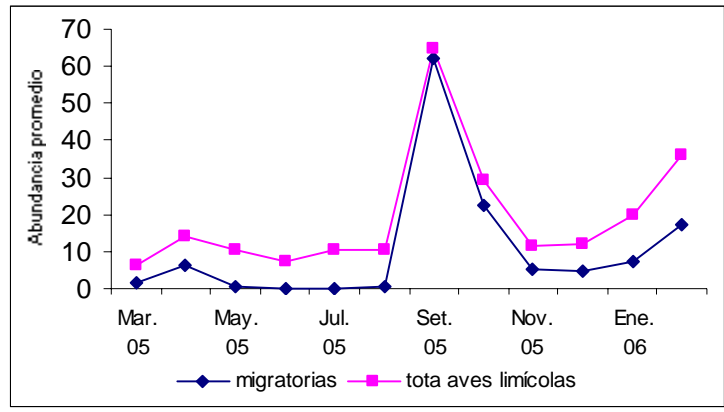

Figura 4. Variación mensual de la abundancia promedio total de aves limícolas y migratorias en la laguna Marvilla y orilla de mar.
Para ésta zona, la especie más abundante fue el playero blanco Calidris alba. En 1987, Castro y Myers en un trabajo de investigación llevado a cabo en la orilla de mar considerada dentro del Refugio de Vida Silvestre Pantanos de Villa reportaron la presencia de una población de 800 a 1000 individuos en el mes de Octubre, la cual se mantenía hasta Abril disminuyendo gradualmente a medida de que estos empezaban su migración. Así mismo, reportaron censos para esta especie de años anteriores en donde resaltan el alto número de individuos que se podía encontrar en la costa peruana. En nuestro caso, a pesar de ser ésta la especie más abundante esta sólo fue registrada entre Septiembre y Noviembre y su población osciló entre los 20 y 73 individuos. Esto nos lleva a plantear la hipótesis de que la población del playero blanco para Pantanos de Villa ha disminuido, sin embargo esto sólo debe ser considerado en la medida que se disponga de mayor información temporal y espacial de esta especie para su comparación.

Diversidad de las aves limícolas en las zonas evaluadas.

Los resultados obtenidos de los valores de diversidad para ambas zonas muestreadas se muestran en la Figura 5. En la zona limosa de la laguna La Herradura el valor mas alto de diversidad fue registrado en el mes de Setiembre con 2.98 bits/ind., mientras que los valores mas bajos se registraron en Junio y Julio con 1.0748 bits/ind. y 1.3740 bits/ind. respectivamente, fecha que corresponden a la época en que la mayoría de las aves migratorias no se encuentra presente en el Hemisferio sur. En la Laguna Marvilla y orilla de mar a diferencia de lo registrado en la zona limosa de la laguna La Herradura, los índices de diversidad son bastante bajos siendo el valor más alto 1.808 bits/ind. y el más bajo 0.071 bits/ind. Es importante señalar que en Junio no se pudo obtener ningún valor ya que solo se observó una sola especie, Charadrius vociferus.

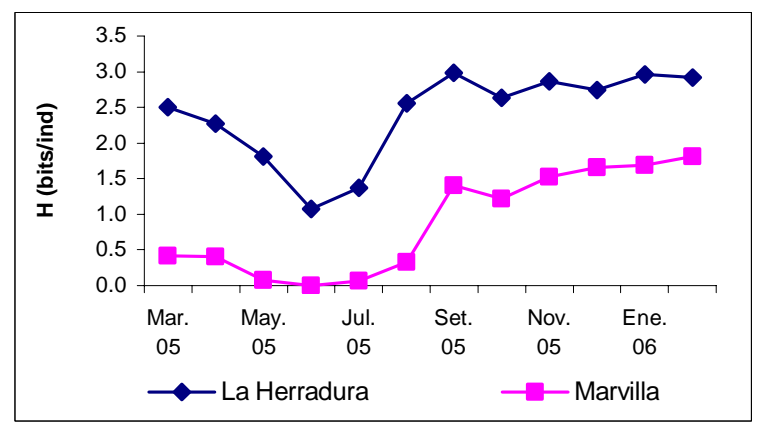

Figura 5. Valores de diversidad a lo largo del año muestreado - Zona limosa laguna la Herradura y laguna Marvilla y orilla de mar. 
La zona limosa de la laguna La Herradura ostenta valores más altos de diversidad que los obtenidos en la laguna Marvilla y orilla de mar. En ambas zonas el número de especies fue muy cercano, pero la abundancia de estas fue diferente. Esta variación en la abundancia respondería a las características del sustrato, limo y arena, y a la disponibilidad de alimentos en ambos lugares lo que repercutiría directamente en la diversidad (Torres, 2006).

A pesar de que las especies registradas en ambas zonas muestreadas fueron en su mayoría las mismas, el uso que hacen éstas del hábitat fue diferente. La mayor parte de aves limícolas utilizan la zona limosa de la laguna La Herradura para forrajear y descansar y solo algunas se movilizan a la laguna Marvilla a descansar y en menor medida a forrajear. A pesar de la menor diversidad de aves en la laguna Marvilla, esta zona es importante por presentar otro tipo de hábitat utilizado por las aves propiamente de orilla como Calidris alba.

Finalmente, de las observaciones realizadas mensualmente a lo largo del año evaluado se apreció que al no existir un sistema de drenaje y manejo del agua, la zona de limo fue inundada en algunas oportunidades, lo cual perjudicó seriamente a las aves limícolas de menor tamaño que utilizan exclusivamente los bordes de las zonas de limo en su alimentación y desplazamiento (Torres, 2006). Por lo tanto, es indispensable definir un programa de manejo de aguas, así como de protección de las zonas utilizadas por estas aves, lo que garantizará su protección y conservación.

\section{Conclusiones}

En el Refugio de Vida Silvestre Pantanos de Villa se reportaron durante el período 2005 - 200618 especies de aves limícolas, 15 de ellas migratorias y 3 residentes.

La zona limosa de la laguna La Herradura presentó la mayor abundancia promedio dada principalmente por las aves migratorias. Esta abundancia estuvo relacionada con el tipo sustrato presente en la zona (limo) y la composición de los invertebrados macrobentónicos. Se registró dos meses de mayor abundancia uno en Setiembre y otro en Enero; el aumento en este último mes sugeriría que poblaciones de algunas aves migratorias de otros humedales al retornar a sus lugares de reproducción utilizarían a Pantanos de Villa como un lugar de parada dentro de su ruta migratoria.

La zona limosa de la laguna La Herradura obtuvo un índice de diversidad mayor a la obtenida en la laguna Marvilla y orilla de mar, como resultado de la mayor abundancia de aves limícolas presentes en la primera zona. Sin embargo la laguna Marvilla y la orilla de mar son zonas importantes por presentar otro tipo de hábitat para las aves de orilla.
El manejo inadecuado del agua puede originar la pérdida de la zona de limo poniendo en riesgo a las aves migratorias que hacen uso de este lugar, las cuales pueden ser consideradas aves emblemáticas de este tipo de hábitat.

\section{Agradecimientos}

Deseo agradecer a las personas encargadas del manejo del Refugio de Vida Silvestre Pantanos de Villa por facilitar la realización de este estudio, así como también a todas las personas que colaboraron conmigo en la obtención de los datos de campo. Un agradecimiento especial a Daniel F. Lane, Irma Franke, Arturo Carlos y Edgar Sánchez por sus valiosos aportes y sugerencias.

\section{Literatura citada}

Baldassarre G. \& Fischer D. 1984. Food Habits of Fall Migrant Shorebirds on the Texas High Plains. J. Field Ornithol. 55(2): 220-229.

Barbosa A. \& Moreno E. 1999. Evolution of foraging strategies in shorebirds: an ecomorphological approach. Auk. 116(3): 712-725.

Berthold P. 1993. Bird Migration: A General Survey. Oxford University Press nc., New York.

Blanco D. 2000. Los Humedales como Hábitat de aves acuáticas. Boletín UNESCO, Uruguay. : 208-217.

Canevari P., Castro G., Sallabery M.\& Naranjo L. 2001. Guía de los Chorlos y Playeros de la Región Neotropical. American Bird Conservancy, WWF- US, Humedales para las Américas y Manomet Conservation Science, Asociación Calidris. Santiago de Cali, Colombia.

Castro G. \& Myers J. 1987. Ecología y conservación del playero blanco (Calidris alba) en el Perú. Boletín de Lima. (52): 47- 60.

Castro G., Myers J. \& Bertochi L. 1988. Predadores del "playero blanco" en el Perú. Boletín de Lima. (58): 5759.

Clements J. \& Shany N. 2001. A Field Guide to the Birds of Peru. Temecula: Ibis Publishing Company.

Eiseimann K. 2006. Evaluation of waterbirds populations and their conservation in Guatemala. Waterbirds Conservation for the Americas.

Eldridge J. 1992. Waterfowl Management handbook. 13.2.14 Management of Habitat for breeding and migration shorebirds in the Midwest. Fish and Wildlife Leaflet 13.2.14. Minneapolis.

Fjeldsa J. \& Krabbe. 1990. Birds of the High Andes. Zoological Museum. Svendborg, University of Copenhagen and Apollo Books, Denmark.

Guerra C. 2004. Distribución espacial - temporal y comportamiento de aves playeras en el Parque Nacional Natural Sanquianga (Nariño, Colombia). Tesis para optar el título de biólogo Universidad del Atlantico Barranquilla.

Guillén C. \& Barrio J. 1994. Los Pantanos de Villa y sus Aves. Boletín de Lima. XVI (91-96): 53-58.

Green A. \& Figuerola J. (s/f) Aves acuáticas como bioindicadores en los humedales. -Documento de la Diputación de Almeida - España. : 47-60.

Hollamby S., Afema-Azihumi J., Waigo S., Cameron K., Gandolf A., Norris A. \& Sikarsie J. 2006. Suggested 
Guidelines for Use of Avian Species as Biomonitors. Environmental Monitoring and Assessment .118(1-3).

INRENA. 1998. Plan Maestro Pantanos de Villa. Refugio de Vida Silvestre. Ministerio de Agricultura. Lima.

Koepcke M. 1964. Las aves del departamento de Lima. Edit. M. Koepcke. Perú.

Kusler J., Mitsch W. \& Larson J. 1994. Humedales. Investigación y Ciencia. 210: 6-13.

Lyons J. \& Haig S. 1997. Fat content and stopover ecology of spring migrant semipalmated sandpipers in South Carolina. The Condor. 97: 427-437.

Magurran A. 1988. Ecological Diversity and its Measurement. Croom Helm. London.

Myers J., Maron J. \& Sallaberry M. 1985. Going to extremes: Why do Sanderlings Migrate to he Neotropics? Neotropical Ornithology 36. : 520-535.

Pautrat L. \& Riveros J. 1998. Evaluación de la Avifauna de los Pantanos de Villa, Lima. Los Pantanos de Villa, Biología y Conservación. Museo de Hist. Natural UNMSM. Serie de Divulgación. (11): 85-95.

Pulido V., Jahncke J., Nakamatsu P. \& Flores C. 1996. Conservación de los charadriiforrmes en la costa peruana. Shorebirds Ecology and Conservation in the Western Hemisphere. Internacional Wader Studies 8. Presentado al IV Congreso de Ornitología Neotropical. : 55-61.

Pulido V. 1998. La Zona Reservada de los Pantanos de Villa en el contexto de la conservación de los humedales en el Perú. Los Pantanos de Villa, Biología y Conservación.
Museo de Hist. Natural - UNMSM. Serie de Divulgación. (11): 147-159.

Pulido V. 2003. Influencia de la pérdida de hábitats en la conservación de las aves de los Pantanos de Villa. Tesis para optar el grado Académico de Doctor en Ciencias Biológicas. UNMSM.

RAMSAR. 2002. UICN. Oficina Regional para Mesoamérica. Convención Internacional relacionada con los Humedales y el Medio Marino de Mesoamérica. UICN 1 edic. Costa Rica. : 112.

Recher H. \& Recher J. 1969. Some Aspect of the Ecology of Migrant Shorebirds. II Agression. Wilson Bulletin. 81 (2).

Rundle D. 1982. A case for esophageal analysis in shorebirds food studies. J. Field Ornithol. 53(3): 249257.

Tabilo E. 2003. El beneficio de los humedales en la Región Neotropical. Centro Neotropical de Entrenamiento en Humedales, La Serena - Chile. : 73.

Torres M. 2006. "Dinámica temporal de la comunidad de aves (Haematopodidae, Charadriidae, Scolopacidae, Recurvirostridae y Phalaropodidae) en la Zona Reservada de los Pantanos de Villa durante el año 2005” Tesis para optar el título de Bióloga. Universidad Nacional Agraria - La Molina.

Wust W., Luscombe A. \& Valqui T. 1994. Las Aves de los Pantanos de Villa y Alrededores. Asociación de Ecología y Conservación (ECCO), Lima.

${ }^{1}$ Departamento de Ornitología. Museo de Historia Natural - UNMSM, Av. Arenales 1256 - Jesús María. Lima Perú. E- mail: miriamtorresn@yahoo.es

${ }^{2}$ Laboratorio de Ecología de Procesos, Dpto. de Biología, Universidad Nacional Agraria La Molina Lima - Perú. Av. La Molina s/n La Molina, Lima - Perú. Apartado Postal 12-056 Lima 12 Perú. 